\title{
Occupational Stress: The Role of Psychological Resilience in the Ecological Transactional Model
}

\author{
Ruben-Wen Sivam, MA \\ Triskelion Group, Singapore \\ Weining C. Chang, PhD, Adjunct Prof. \\ Research in Clinical Sciences, Duke-National University of Singapore \\ Graduate Medical School, Singapore
}

doi: 10.19044/esj.2016.v12n14p63 URL:http://dx.doi.org/10.19044/esj.2016.v12n14p63

\begin{abstract}
The present study adopts an ecological approach to examining occupational stress, which incorporates both a trait and a transactional perspective to offer a more comprehensive conceptualization of the antecedents and outcomes of stress with psychological stress as a moderator. Data collected from 182 job incumbents supported the hypothesized relationships among primary appraisal, secondary appraisal, psychological resilience, and maladaptive outcomes. Results from structural equation modeling revealed that primary appraisal affected secondary appraisal, and secondary appraisal, in turn, contributed to maladaptive outcomes. In addition, the relationship between primary appraisal and maladaptive outcomes was partially mediated by secondary appraisal. Consistent with our hypothesis that resilience plays an adaptive role in the stress process, the results indicated that resilience had a direct influence on secondary appraisal. Resilience also affected maladaptive outcomes of stress but this relationship was partially mediated by secondary appraisal.
\end{abstract}

Keywords: Occupational Stress, Psychological Resilience, Asian Context

\section{Introduction}

The rapidly changing nature of work in the $21^{\text {st }}$ century poses challenges for both the organization and the individual worker. We are living in a new economy - powered by technology, fueled by information, and driven by knowledge. Increasing pressures on organizations to be more competitive, agile, and consumer focused has generated much interest in the "lean enterprise" that first captured attention in manufacturing. In today's global economy, the adoption of lean principles and thinking, propelled by rapid technological advancement, has led to a multitude of changes in 
organizational structure to improve the efficiency of internal processes, with a goal of eliminating waste and redefining customer value. This necessitates that employees acquire new proficiencies or modify existing behaviors to be competitive for different jobs (Kinicki \& Latack, 1990) and be globally effective across a variety of geographies and cultures (Noe \& Ford, 1992). These trends in the global work environment have resulted in increased pressure on employees to take on a more competitive approach, to adapt to the ever-changing job demands, and to behave in functionally flexible ways.

Such organizational change has been associated with substantial increases in psychological strain due to feelings of loss and job insecurity (Cartwright \& Cooper, 1990). The increasing emphasis on the importance of understanding occupational stress at both the individual and the organizational level is a corollary of the rising cost incurred by organizations and employees (e.g., Vecchio, Hearn, \& Southey, 1996). The World Health Organization (WHO) estimates the cost of stress to organizations in the United States in the region of USD $\$ 300$ billion per year, with a myriad of health problems faced by employees, such as heart disease and a multitude of mental health issues often linked to work-related stress (e.g., Alfredsson \& Theorell, 1983).

Moreover, the more recent worldwide economic downturn has brought in an exigency for a clearer conceptualization of the occupational stress process, and its consequences on health and mental health. This need for a better understanding on how people weather occupational stress is particularly acute in Asian countries - where we are experiencing unprecedented economic development that requires fundamental changes in people's daily lives and work-related behaviors. Furthermore, we need to identify protective factors that moderate the relationship between occupational stress and its damaging impact on physical and mental health that would fundamentally hamper the individual's wellbeing and productivity.

Our research explicates an ecological approach (Atkinson \& Violato, 1994; Moos \& Schaefer, 1993) to examining occupational stress in which both cultural and personal influences on the stress process are considered. Drawing on a cognitive-relational theory of stress (Lazarus \& Folkman, 1984), cognitive appraisals, maladaptive outcomes of stress, and proposed resilience factors within an Asian context were measured and a hypothesized model developed in order to evaluate the functional relations among these variables. 


\section{Theoretical Perspectives on Stress}

\section{Trait Perspective}

Contemporary stress research grew out of an older, psychodynamicoriented literature that emphasized the importance of ego defenses as mechanisms for adapting to stress and anxiety, which led to the emergence of trait-theories of stress (Suls et al., 1996). Empirical support for the ego defense perspective consisted largely of case studies and failed to provide a distinction between defense mechanisms and outcomes (Suls et al., 1996). Furthermore, measurements of coping traits were found to be relatively poor predictors of behavioral and affective responses in specific adaptation contexts (Cohen \& Lazarus, 1973; Watson \& Hubbard, 1996).

The Transactional Model

The limitations of trait approaches led to a paradigmatic shift towards more cognitively and process-oriented slants that offered greater flexibility in their conceptualization of stress as a specific transaction between the person and the environment (Watson \& Hubbard, 1996). The transactional perspective emphasizes the process rather than traits, defining stress as a result of an interaction between the person and the environment. Transactional theorists fundamentally agree that there are four key components in the stress process: the stressor, the environment, the person, and the outcome; and it is the interaction between the stressor, the environment and the person that brings about the phenomenological experience of stress (e.g., Lazarus, 1966; McGrath, 1970).

Based on cognitive-relational theory, Lazarus and Folkman (1984) posit cognitive appraisals as the mediator between the stressor and stressrelated adaptive outcomes; this approach is both relational and process oriented (Folkman, 1984). The theory's relational characteristic is evident in the definition of stress as a transaction between the person and the environment. Stress is not viewed as an inherent property of either the person or the environment, but rather, as a particular relationship between person and environment, that is, the person-environment interaction.

The process-orientation of the theory has two implications: firstly, that the person and the environment are in a reciprocal dynamic interaction, with the person and the environment acting on each other (Folkman, 1984).

Within the theoretical formulation offered here, two major forms of cognitive activities that converge to shape the meaning of every encounter have been postulated- primary and secondary appraisals (Lazarus \& Folkman, 1984).

Primary appraisal. Primary appraisal refers to the process through which the person evaluates the significance of a specific environmental event with respect to the wellbeing of the self and others. It refers to judgments that a transaction is irrelevant, benign-positive, or stressful. If it is judged 
relevant and offsets the homeostatic balance of the individual, the event is considered a stressor (Lazarus \& Folkman, 1984).

Secondary appraisal. Secondary appraisal refers to the evaluation of the coping resources and options available to the individual to manage the stressor-person transaction (Lazarus \& Folkman, 1984). Such appraisals come into play when an encounter is perceived as threatening and involves situational evaluation of potential of control over the transaction, whereby physical, social, psychological, and material assets are evaluated with respect to the person-event transaction (Lazarus \& Folkman, 1984).

Coping. As outlined in the transactional framework (Lazarus \& Folkman, 1984), coping is defined as a person's attempt to manage the transaction by changing affective, cognitive, or behavioral efforts to manage specific external and/or internal demands that are appraised as exceeding the person's resources (Lazarus \& Folkman, 1984). The model delineates two general types of coping: problem-focused coping and emotion-focused coping.

Lazarus and Folkman (1984) posit that primary appraisals of what is at stake and secondary appraisals of coping options are crucial components of the stress process in that they interact with each other to shape the degree of stress and the magnitude of the maladaptive reactions. The individual and the environment are thus seen as having a reciprocal impact on each other, which places proper emphasis on the analysis of stress in terms of personenvironment interaction or transaction.

Ecological Approach: Towards Integration of Trait and Transactional Perspectives

More recent theoretical developments in stress and coping have emphasized the importance of including person factors together with the contextual and situational factors to derive a more comprehensive formulation of the stress-and-coping process (Suls et al., 1996). Empirical evidence suggests that personality is a reasonably reliable predictor of stress and coping (Terry, 1994). Carver et al. (1989) found low to moderate correlations between participants' dispositional coping styles and subsequent reports of situation-specific coping efforts. Holahan and Moos' (1987) study indicated that the initial measures of coping accounted for a significant proportion of variance in subsequent coping responses assessed a year later. Further, McCrae (1989) found evidence of stability in people's coping responses across a seven-year time span. Such findings have underscored the significance of the inclusion of person or individual difference variables in the prediction and etiology of stress.

In addition, cultural values can exert a substantial influence on an individual's response to stress. The influence of culture on stress and coping has been well documented in the literature (e.g. Cross, 1995; Kawanishi, 
1995). O’Brien and DeLongis (1996) argue that the role of personality in stress may, in part, depend on the context in which the stressor occurs. Specifically, cultural influences may impinge on the personality variables that play a role in the stress process (Suls et al., 1996).

These developments have resulted in a general consensus among theorists over fundamental issues concerning stress-and-coping: stress cannot be explained by the transactional perspective or person factors alone. Instead, a synthesis of the two perspectives offers a holistic and more comprehensive outlook that facilitates understanding of the antecedents and outcomes of stress (Goh, 2003).

As such, contemporary theorists have conceptualized stress in terms of an ecological model (Atkinson \& Violato, 1994; Moos \& Schaefer, 1993). Lazarus (1991) utilized the term 'adaptive-fit' to articulate a key feature of this model: a favorable person-environment fit is crucial to positive outcomes. Negative outcomes, in this context, are a result of incongruence between an individual's needs, actions, or aspirations, and current situational constraints. This approach enables conceptualization of components of the stress process as part of a holistic system, in which positive outcomes are the result of a good fit between the variables.

Adopting an ecological model presents a prospect to reconcile differences between personality and transactional approaches to stress. This perspective is of central importance because it forms the methodological and conceptual foundation of the present study, in which both personality and transactional approaches to stress are integrated within an Asian cultural context.

The following section outlines the resilience factors (person factors) that are posited to play a role in the stress process.

\section{Psychological Resilience in the Contemporary Asian Context \\ The Construct of Resilience}

The construct of resilience in contemporary psychological literature refers to a dynamic process encompassing positive adaptation within the context of significant adversity. Following Werner's groundbreaking study on children in Hawaii (Werner, Bierman, \& French, 1971), research on psychological resilience has expanded to include multiple adverse conditions such as socioeconomic disadvantage, maltreatment, urban poverty, and catastrophic life events (e.g., Beeghly \& Chicchetti, 1994; Garmezy, 1991; Luthar, 1999; O’Dougherty-Wright et al., 1997; Werner \& Smith, 1992).

While early efforts were primarily focused on personal qualities of resilient individuals, such as high self-esteem or autonomy (Masten \& Garmezy, 1985), the focus of more contemporary empirical work has shifted 
from identifying the protective factors to evaluating the dynamics of the underlying processes (Cowen et al., 1997; Luthar, 1999).

Recent investigations into mental health and adjustment to stress tend to focus on cognitive factors that underlie the construction of the phenomenological experience of the self and the world. Such inquiries suggest that individual differences in these cognitive factors will determine whether an individual perceives environmental stressors as barriers or challenges. For instance, dysfunctional cognitive styles, such as pessimistic attribution (Abramson, Seligman, \& Teasdale, 1978), are thought to be important because of their mediating role between negative events and adverse physical and mental health outcomes (Abramson, Seligman, \& Teasdale, 1978; Seligman et al., 1979). An impressive body of empirical studies has identified relationships between these cognitive constructs and positive adjustment of individuals in Western populations (e.g., Abramson, Seligman, \& Teasdale, 1978; Peterson \& Seligman, 1984; Seligman et al., 1979).

Research on Asians

Towards the the turn of the millennium, research suggests that, relative to their Western counterparts, Asians have higher levels of maladaptive or dysfunctional cognitive styles, such as, a relatively external locus of control (Bond \& Tornatzky, 1973), lower optimism (Heine \& Lehman, 1995), and lower global individual self-esteem (Heine et al., 1999). Evidence also suggests that Asians tend to be more willing to accept information indicative of their failures (Heine, Takata, \& Lehman, 2000), are more likely to recall events regarding negative information (Meijer, Hiene, \& Yamaguchi, 1999), and their self-evaluations are more affected by failures than successes (Kitayama et al., 1997).

Paradoxically, in spite of these vulnerability factors, which suggest that Asians should be wrought with stress, depression, and other maladjustment symptoms, research has shown that the prevalent rates of depression are actually lower in Asian populations as compared to Western ones (Chang, 1985). Whether a psychological process produces positive or negative effects on the individual, depends on the ecological and cultural context of the individual. Studies have yielded evidence to support the notion of the positive effect of collectivist attitudes in alleviating loneliness (Neo, Chang, \& Fung, under review) and promoting collective well-being in the collectivist context (Chang, Chu, Tong, and Koh, 2002). While the belief that the individual has little control over the event may signal negative adjustment, in situations where the individual realistically has little control over the event, acceptance of the situation would signal better adjustment (Chang,Toh, Chen, \& Fan, 2016). Such empirical findings suggest that there 
might be different person factors mediating between vulnerability and adjustment outcomes in the Asian context.

Resilience Factors in the Contemporary Asian Context

It is postulated that resilience is a dynamic process involving multiple constructs in the individual that might act as a buffer between stressors and the self. Chang and Lim (2007) have conceptualized resilience in the Asian context as a coherent three-dimensional construct. The three facets of psychological resilience are (a) malleable belief about the self, (b) emotional self-regulation, and (c) coping flexibility.

Malleable belief about the self. In an Asian context, the self is considered an ever-evolving, dynamic, and flexible agent, and the project of life is a proactive process, leading from the isolated independent self to a self that is dynamically integrated with the community (Tu, 1985). Growth and maturity of the self is in flexible integration with others rather than separation from the collective.

Within this context, a factor that might help the individual cope with stress would involve a sense of self-efficacy in terms of seeking integration with the environment, rather than conquering it, work with the environment to achieve the most productive outcome. In this context, environmental challenges are seen as the opportunity for further growth of the individual; in time of adversity, the individual thus seeks to develop within and with the community.

Along the same line of argument, Heine (2001) argues that the cultural task of fitting in and achieving interdependence leads to a belief in the improvability of the self. Hence, rather than distancing oneself from a situation where one fails or perceives obstacles to one's goals, Asians are encouraged to meet failure with increased effort. Encounters with failure should not signal permanent inadequacies of the self, leading to selfprotective or ego-defensive strategies. Instead, such individuals would see obstacles and frustrations encountered as challenges for learning and further development, and should show more vigorous effort in response to impediments (Dweck, 2000). The malleable belief about the self thus enables the individual to be flexible in times of change.

Emotional self-regulation. Emotional self-regulation is defined as the individual's effort to regulate one's own emotions and to manage emotional behaviors. In the stress and coping literature, emotion-focused coping focusing on management of emotions as a way of coping has been identified as one major category of coping reactions (Lazarus \& Folkman, 1984). Although emotional self-regulation is often attributed to the executive functions of the brain, in every culture, there are tried-and-true ways of regulating one's emotions. Such beliefs and strategies are coping resources 
originated from the world-views and philosophies that model the personsituation relationships or the control beliefs of the individual.

Rothbaum et al.'s (1982) primary-secondary model defines primary control as the belief that individuals can enhance their welfare by influencing existing realities; and secondary control as the belief that individuals can enhance their welfare by accommodating to existing realities by way of changing one's cognition, affect and/or behavior. With a preference for secondary control beliefs (Chang, Chua \& Toh, 1997), Asians would be more ready to accept an uncontrollable situation and gain peace and serenity in the face of loss and failure. This has been found to be a salient characteristic of emotional regulation efforts in many Asian populations (Chang, 2007).

Logically, it follows that emotional self-regulation, which is a key process through which secondary control manifests, should play a significant role in mediating between stressors and adaptive outcomes.

Coping flexibility. Coping flexibility refers to an underlying mechanism that regulates the use of different coping strategies in order to achieve a person-situation fit. Given that in Asian collectivist societies, the self is viewed as interdependent with the surrounding context; and it is the self-in-relation-to-other that is the focal point in individual experience (Markus \& Kitayama, 1991), individuals tend to adhere to a contextual theory of behavior. Empirical evidence substantiating this conception suggests that Asians, as compared to their American counterparts, are more likely to take situational information into account (Heine, 2001). These aspects of the Asian self point to values and expectations that will favor a situation-sensitive attribution style. Empirical support for coping flexibility in Asians is evident from Hong and Chang's (2002) study, which found that Asians tended to use more varied coping strategies, and were attentive and responsive to the perceived demands of the situation.

It follows that in a modern Asian society where there are complex roles and situations to which the individual has to adapt, coping flexibility might be adaptive to the extent that it facilitates fit with the situation. Thus, coping flexibility is conjectured to be a fundamental constituent in the resilience process.

\section{Resilience in the Cognitive-Relational Framework of Occupational Stress}

The primary purpose of the present study is to facilitate understanding of the nature of resilience factors in the Asian context and their relationship to the cognitive-relational stress process. The hypothesized model for resilience in the occupational stress process is presented in Figure 1. 
The Effect of Primary Appraisal on Secondary Appraisal

According to Lazarus and Folkman's (1984) transactional theory, at the primary appraisal stage, the individual determines if an encountered event is benign/positive, irrelevant, or stressful. If the encountered event is perceived as stressful, the secondary appraisal process is triggered. The two appraisal processes are closely related such that assessments made during primary appraisal have an effect on secondary appraisal. Empirical support for this theoretical contention comes from a study on the occupational stress process which found a significant negative relationship between primary and secondary appraisals (Goh, 2003). Consistent with the transactional perspective, we hypothesized that primary appraisals of the significance of an event with respect to well-being will have a negative effect on secondary appraisals of control. That is, individuals who perceive an event as threatening and relevant to the self are more likely to perceive less controllability with respect to the encounter.

Hypothesis 1: Primary appraisal has a direct negative effect on secondary appraisal.

The Effect of Secondary Appraisal on Maladaptive Outcomes

The transactional perspective (Lazarus \& Folkman, 1984) posits that secondary appraisals of controllability within an encounter shape the strength and quality of outcomes. For example, all other things being equal, if a person perceives helplessness in dealing with a demand, stress will be relatively great because the harm/loss cannot be overcome or prevented (Lazarus \& Folkman, 1984).

It is noteworthy that the concept of coping is interlinked with the appraisal process. Monroe and Kelley (1997) argue that while secondary appraisal is affected by the individual's perceived ability to cope with the event, over time the actual coping activities and their efficacy influence the appraisal process itself. Yet theoretically, it is important to distinguish the concept of coping from that of appraisal. Thus, we focus on the interplay between the appraisal processes, psychological resilience and the outcome in the stress-and-coping process.

Empirical investigations have indicated a direct link between secondary appraisal and outcomes of stress. Folkman et al.'s (2001) study on the coping processes of couples indicated a strong and direct relation between two measures of secondary appraisal and the satisfactory level of the outcomes, which have an effect on the psycho-physiological experience of the encounter. In his attempt to clarify the etiological process of occupational stress and coping, Goh (2003) had job incumbents respond to appraisal, coping and stress measures over a period of between two to four weeks. Using path-analysis, the results established that secondary appraisals directly and significantly predicted stress outcomes. Researchers have argued 
that irrespective of the objective level of control, believing that one has control over adverse events is, in itself, stress-reducing and believing one has little or no control over them is stress-inducing (e.g., Schaubroeck \& Merritt, 1997; Spector, 1986). Hence, secondary appraisal is expected to have a direct negative effect on maladaptive outcomes.

Hypothesis 2: Secondary appraisal has a direct negative effect on maladaptive outcomes.

The Effect of Primary Appraisal on Maladaptive Outcomes

As predicted above, individuals’ primary appraisals of an event are expected to influence secondary appraisals, and secondary appraisals of the stressor are, in turn, expected to influence maladaptive outcomes. In addition to this indirect effect of primary appraisal on maladaptive outcomes, there is empirical evidence to support that primary appraisal has a direct effect on stress. In a study on the etiological process of occupational stress, Goh (2003) found a direct path from primary appraisal to stress outcomes.

If an individual has a high stake in the outcome, helplessness is potentially devastating; and even when people believe that they have considerable power to control the outcome of an encounter, perceptions of high stakes can produce considerable stress (Lazarus \& Folkman, 1984). Folkman (1984) posits that given a situation in which a stressful encounter is appraised as controllable, two different antecedent primary appraisals of the encounter are possible: threat and challenge. A challenge appraisal should produce more positive outcome because it facilitates effective problemfocused coping and promotes good morale. In contrast, a threat appraisal may impede problem-focused coping efforts thus leading to poor problem resolution and negative outcomes.

As shown in Figure 1, primary appraisal was expected to have a direct positive effect on maladaptive outcomes. The direct effect from primary appraisal to maladaptive outcomes is one of the two hypothesized constituents of the total effect of primary appraisal on maladaptive outcomes. The other constituent of the total effect is the indirect influence of primary appraisal on maladaptive outcomes through secondary appraisal of the event.

Hypothesis 3: The effect of primary appraisal on maladaptive outcomes is partially mediated by secondary appraisal.

The Effect of Resilience on Secondary Appraisal

Basically, the proposed resilience factors are significant because they may affect appraisals of an event or stressor. Previous research findings on the impact of personality factors on stress have shown that individuals with high Neuroticism tend to have high negative affects, and are prone to appraise situations negatively (Atkinson \& Violota, 1994; David \& Suls, 1999). Individuals high on extraversion, on the other hand, have been found to measure highly on positive affect, and tend to have more positive 
appraisals of situations (David \& Suls, 1999; Meyer \& Shack, 1989). To the extent that the resilience factors augment perceptions of impediments as opportunities to learn, dynamic flexibility in approaching stressful events, and active control over the self, it is conceivable that they may facilitate appraisals of controllability. Resilient individuals may perceive and actually have a wider repertoire of resources within the self to deal with an aversive event. As efficacy expectancies increase and the person judges his or her resources more adequate for satisfying task demands, the relationship is evaluated as holding the potential for more control (Folkman, 1984).

The psychological mechanism responsible is hypothesized to involve the influence of resilience factors on preexisting notions about reality, which serve as a perceptual lens to determine "how things are" with respect to an event and shape a person's perception of his or her relationship to the environment (Lazarus \& Folkman, 1984). Hence, we hypothesize that resilience has a direct positive effect on secondary appraisal.

Hypothesis 4: Resilience has a direct positive effect on secondary appraisal.

\section{The Effect of Resilience on Maladaptive Outcomes}

As mentioned, resilience is posited to play an adaptive role in the stress process insofar as it fosters responsiveness to the perceived demands of the situation and a sense of self-efficacy in terms of controlling oneself, which would heighten one's psychological well-being. Resilient individuals tend to view impediments encountered as challenges for learning and further development, rather than indicators of inadequacies of the self. As a result, the proposed resilience factors may facilitate more effective and adaptive coping strategies in dealing with stress. For example, coping flexibility regulates the use of both primary and secondary control coping strategies and facilitates flexibility in dealing with a particular event (Hong \& Chang, 2002); and emotional self-regulation fosters a sense of self-efficacy in terms of controlling the self, which would reduce negative emotional and psychological reactions to a stressor (Yamaguchi, 2001).

Consistent with this perspective, it is expected that individuals with higher levels of resilience would experience lower levels of anxiety, depression, and negative affect. Hence, as depicted in Figure 1, we expected a significant negative direct effect from resilience to maladaptive outcomes. The direct effect of resilience on maladaptive outcomes is one of the two hypothesized constituents of the total effect of resilience on maladaptive outcomes. The other constituent of the total effect is the indirect effect from resilience to maladaptive outcomes through secondary appraisal. As previously posited, relative to those low in resilience, individuals with higher levels of resilience are more likely to perceive greater controllability within 
the encounter and it is this secondary appraisal that has a direct effect on reducing maladaptive outcomes of stress.

Hypothesis 5: The negative effect of resilience on maladaptive outcomes is partially mediated by secondary appraisal.

\section{Method}

$\underline{\text { Participants }}$

A total of 188 job incumbents from a local (Singapore) branch of a multi-national corporation were recruited for the present study. Of the total sample, 182 provided usable data. Two participants were found to be outliers and four provided incomplete data; they were excluded from all data analyses. The usable sample was composed of 103 males and 79 females, with an age range of 24-60 (mean $=40, \mathrm{SD}=8.80$ ). All the participants were Singaporean (with ethnic representation similar to the Singapore citizen population), had at least an O-level education, and were proficient in English. The sample came from a variety of organizational departments such as finance, human resource, base operations, area management, and fleet.

\section{Measures}

Primary and secondary appraisal measure. The Stress-AppraisalMeasure (Peacock \& Wong, 1990) was adapted to assess both primary and secondary appraisals. Participants were required to indicate the extent to which they agreed with the given statements with respect to a stressor on a 5point scale with anchors ranging from 1 (strongly disagree) to 5 (strongly agree). The primary appraisal measure consisted of three dimensions relevant to anticipatory stress, namely, threat, challenge, and centrality. Threat appraisals involve the potential for harm/loss in the future, and challenge appraisals reflect the anticipation of gain or growth from the experience. Centrality refers to the perceived importance of an event for an individual's well-being, and is conceptually similar to the idea of stakes (Peacock \& Wong, 1990). The primary appraisal measure consisted of 12 items in total, with each dimension represented by four items. Example items under the threat sub-scale were "The evaluation is threatening for me," and "The evaluation has negative impact on me.” Example items under the centrality sub-scale included "The evaluation has important consequences for me," and "The evaluation has serious implications for me." The challenge sub-scale was represented by items such as "The evaluation has positive impact on me," and "I am excited about the outcome of the evaluation." Cronbach's alphas for the threat, centrality, and challenge sub-scales were .88, .91, and .79 respectively in the present study.

The secondary appraisal measure focuses on perceptions of control (Peacock \& Wong, 1990). It delineates individual assessment of controllability in terms of three dimensions: the extent to which an event is 
controllable by self, controllable by others, and uncontrollable by anyone. The secondary appraisal measure consisted of 12 items in total, with each sub-scale made up of four items. Example items represented by the controllable by self dimension were "I have the ability to do well with regard to the evaluation," and "I have what it takes with regard to the evaluation." Example items under the controllable by others sub-scale were "There are resources available to handle the evaluation," and "There is someone who can help me with regard to the evaluation." Finally, items under the uncontrollable by anyone sub-scale included "I feel totally hopeless about the evaluation," and "The outcome of the evaluation is uncontrollable." Cronbach's alphas for the controllable by self, controllable by others, and uncontrollable by anyone sub-scales were $.86, .85$, and .89 respectively.

Resilience measure. The resilience measure (Chang, \& Lim 2007) consisted of the following scales: incremental implicit belief about the self (8 items), emotional self-regulation (8 items), and coping flexibility (12 items). Participants were required to indicate the extent to which they agreed with the given statements on a 5-point scale with anchors ranging from 1 (strongly disagree) to 5 (strongly agree). Incremental implicit belief about the self was assessed using items such as "If one makes a mistake in doing something, one can always learn from one's mistakes", and "One's personality can always be improved". Emotional self-regulation was assessed using items such as "I make independent judgments of myself, regardless of what other people might say or think about me," and "When I find myself worrying, I work on myself to reduce the worries." Example items from the coping flexibility scale included "I am aware to which situations I am able to exercise control and which situations I am not," and "Changing strategies in order to alleviate stress caused by an event is easy for me." Cronbach's alphas for the incremental implicit belief about the self, emotional selfregulation, and coping flexibility sub-scales were .75, .82, and .90 respectively.

State anxiety measure. The State Anxiety Inventory (Spielberger et. al., 1970) was used to assess state anxiety, which is defined as a temporal cross-section in the emotional stream-of-life of a person, consisting of subjective feelings of tension, apprehension, nervousness, worry, and autonomic arousal (Spielberger et. al., 1970). Participants were required to indicate the extent to which they felt what was described by the given statements on a 4-point scale with anchors ranging from 1 (not at all) to 4 (very much so) with higher scores indicative of higher levels of anxiety. Example items from the 20-item scale included "I am presently worrying over possible misfortunes", “I feel strained”, and "I am jittery”. Cronbach's alpha for the State Anxiety Inventory was .93. 
Depression measure. The Zung Self-Rating Depression Scale (Zung, 1965) was used to evaluate the extent of maladjustment. The scale, a traditional measure of depression, required participants to indicate the extent to which they experienced what was described by each statement in the past week on a 5-point scale ranging from 1 (strongly disagree) to 5 (strongly agree), with higher scores indicating greater emotional distress. Example items from the 20-item scale included "My heart beats faster than normal", "I am restless and can't keep still", and "I am more irritable than usual". Cronbach's alpha for the Zung Self-Rating Depression Scale was .77.

Negative affectivity measure. Negative affectivity (NA) is a general dimension of subjective distress and unpleasant engagement that subsumes a variety of aversive mood states, including anger, contempt, fear, and nervousness, with low NA being a state of calmness and serenity. NA was assessed using the Negative Affect Schedule developed by Watson, Clark, and Tellegen (1988). The scale required participants to indicate the extent to which they experienced each affect in the past week on a 5-point scale with anchors ranging from 1 (very slightly or not at all) to 5 (very much). Example items from the 10-item scale included "irritable", "nervous", and "distressed". Watson et al. (1988) reported that the Negative Affect Schedule displayed high degrees of reliability as well as convergent and divergent validity. Cronbach's alpha for the negative affect scale was .94 in the present study.

Procedure

The data was collected during the organization's year-end performance evaluation period. The evaluation was conceived to be a stressor insofar as employees' performance would determine their year-end variable bonus as well as likelihood of promotion. Participants were tested individually in two sessions, which were placed two weeks apart. During the first session, all participants completed the three resilience measures as well as demographic items (gender, age, race, and nationality). During the second session, they completed the primary appraisal measures (threat, centrality, and challenge), secondary appraisal measures (controllable by self, controllable by others, and uncontrollable by anyone), and the three maladaptive outcome measures (anxiety, depression, and negative affectivity), in that order. Participants were specifically instructed to respond to the appraisal measures with respect to the evaluation. Prior to administration of the measures, participants were told that the measures were mete out for research purposes, and that their responses on the items would be kept confidential and would not be recorded in their personal files or used for any personnel decision purposes. At the end of the session, all participants were thoroughly debriefed and thanked for their participation. 
Data Analyses

Given the multidimensional nature of the constructs in the hypothesized model, full structural equation models were employed in data analysis. Multiple indicators were formulated for each of the latent variables represented in Figure 1. For the resilience latent variable, the three indicators consisted of the incremental implicit belief about the self, emotional selfregulation, coping flexibility measures. For both appraisal latent variables, the three indicators each consisted of 4-item scales based on Peacock and Wong's (1990) formulation previously conferred. Primary appraisal was indicated by the threat, centrality, and challenge measures, and secondary appraisal was indicated by the controllable by self, controllable by others, and the uncontrollable by anyone measures. The anxiety, depression, and negative affectivity measures were used as indicators of the maladaptive outcome latent variable.

The hypothesized model was tested using LISREL 8 (Joreskog \& Sorbom, 1993). The chi-square statistic and a variety of model fit indices were used to assess the adequacy of the model: adjusted goodness-of-fit index (AGFI; Joreskog \& Sorbom, 1989), comparative fit index (CFI; Bentler, 1990), nonnormed fit index (NNFI; Bentler \& Bonett, 1980), incremental fit index (IFI; Bollen, 1989) standardized root mean square residual (SRMR; Joreskog and Sorbom, 1986), and root mean square error of approximation (RMSEA; Steiger, 1990).

\section{Results}

The means, standard deviations, intercorrelations, and reliability estimates of the variables are presented in Table 1. Examination of Cronbach's alphas for the indicators shows that they were within acceptable ranges (Cronbach's alphas $>$.74) signifying that the measures possessed adequate internal consistency reliability.

Examination of the bivariate correlations among the variables provided preliminary support for our hypotheses. Primary appraisal was correlated with both secondary appraisal (mean $\underline{r}=-.29$ ) and maladaptive outcomes (mean $\underline{r}=.30$ ), and secondary appraisal was correlated with maladaptive outcomes (mean $\underline{r}=-.46$ ). Resilience was correlated with both secondary appraisal (mean $\underline{r}=.24$ ) and maladaptive outcomes (mean $\underline{r}=$ .38 ), but not related to primary appraisal (mean $\underline{r}=-.07$ ). Subsequently, the hypothesized model was tested directly using structural equation modeling.

Table 2 shows the model fit indices for the structural equation models fitted. The hypothesized model represented a good fit to the current data, $\chi^{2}$ $(49, \underline{\mathrm{N}}=182)=77.78, \underline{\mathrm{p}}<.05, \mathrm{AGFI}=.90, \mathrm{NNFI}=.95, \mathrm{CFI}=.97, \mathrm{IFI}=$ .97, RMSEA $=.05$, SRMR $=.06$. We then specified a more complex alternative model which included a direct effect between resilience and 
primary appraisal. The fit indices associated with this more complex model were $\chi^{2}(48, \underline{\mathrm{N}}=182)=75.68, \underline{\mathrm{p}}<.05, \mathrm{AGFI}=.90, \mathrm{NNFI}=.95, \mathrm{CFI}=.97$, IFI $=.97$, RMSEA $=.05$, SRMR $=.04$.

Although both models provided a good fit to the data, a chi-square difference test indicated that the increase in model fit in moving from the hypothesized model to the more complex alternative model was nonsignificant, $\Delta \chi^{2}(1, \underline{N}=182)=2.10, \underline{p}>.05$. Moreover, the estimated direct effect from resilience to primary appraisal $(\beta=-.13, \mathrm{p}>.05)$ was also nonsignificant. Hence, on the basis of parsimony, we selected the hypothesized model over the more complex alternative model.

The full hypothesized model with its associated standardized parameter estimates is provided in Figure 2. As shown in Figure 2, all factor loadings and path coefficients were statistically significant, $\mathrm{p}<.05$. To investigate the specific hypotheses concerning the relationships among variables, the individual parameter estimates corresponding to each hypothesis were examined.

Effect of primary appraisal on secondary appraisal. Hypothesis 1, which predicted a direct negative relationship between primary and secondary appraisal, was supported by the direct negative effect from primary appraisal to secondary appraisal, $\beta=-.50, \mathrm{p}<.05$,

Effect of secondary appraisal on maladaptive outcomes. Hypothesis 2 stated that secondary appraisal has a direct negative effect on maladaptive outcomes. This hypothesis was supported by the significant path from secondary appraisal to maladaptive outcomes, $\beta=-.57, \mathrm{p}<.05$.

Effect of primary appraisal on maladaptive outcomes. Hypothesis 3 predicted that the relationship between primary appraisal and maladaptive outcomes is partially mediated by secondary appraisal. The significance of both direct and indirect effects of primary appraisal on maladaptive outcomes provided support for the hypothesis. The indirect positive path from primary appraisal to maladaptive outcomes through secondary appraisal was significant, $\beta=.29,(-.50 \mathrm{X}-.57), \mathrm{p}<.05$. There was also a significant direct path from primary appraisal to maladaptive outcomes, $\beta=.16, \underline{p}<.05$. Summation of the dual effects constituted the total effect of primary appraisal on maladaptive outcomes, $\beta=.45,(.29+.16), \underline{p}<.05$.

Effect of resilience on secondary appraisal. Hypothesis 4, which predicted that resilience has a direct positive influence on secondary appraisal, was supported by the significant direct path from resilience to secondary appraisal, $\beta=.45, \mathrm{p}<.05$.

Effect of resilience on maladaptive outcomes. Hypothesis 5 predicted that the negative relationship between resilience and maladaptive outcomes is partially mediated by secondary appraisal. This hypothesis was supported by the significant indirect path from resilience to maladaptive outcomes 
through secondary appraisal, $\beta=-.26,(.45 X-.57), \mathrm{p}<.05$, and the significant direct path between resilience and maladaptive outcomes, $\beta=$ $.46, \mathrm{p}<.05$. Summation of the dual effects constituted the total effect, $\beta=-$ $.72,(-.26+-.46), \mathrm{p}<.05$.

\section{Discussion}

The results supported our hypothesized model and were consistent with specific predictions made about resilience in the Asian context and its relationships to cognitive appraisals and maladaptive outcomes. Job incumbents' primary appraisals of the stressor affected secondary appraisals, and secondary appraisals, in turn, affected maladaptive outcomes. This is congruent with the general structure and direction of influence proposed by the transactional perspective (Lazarus \& Folkman, 1984) in that the greater an individual perceives an event as threatening and relevant, the lower is his or her perception of controllability over it tends to be, which in turn, is related to higher levels of maladaptive outcomes. In addition, the analysis indicated that primary appraisals had a direct effect on maladaptive outcomes. This provides support for our contention that psychological and emotional reactions to an event cannot be the result of appraisals of controllability alone. Assessments of the significance of a stressor in terms of threat, centrality, and challenge also have some direct impact on the outcomes of stress.

The results also indicated that resilience had a direct influence on secondary appraisal in that resilient individuals were more likely to perceive that they had more control and resources in dealing with a stressful event. The purported psychological mechanism involved the influence of resilience factors on preexisting notions about reality, which serve as a perceptual lens to determine "how things are" with respect to an event and shape a person's perception of his or her relationship to the environment (Lazarus \& Folkman, 1984).

Resilience was found to constitute an important determinant of maladaptive outcomes of stress. The negative relationship between resilience and maladaptive outcomes was partially mediated by secondary appraisal. In addition to the indirect effect of resilience on maladaptive outcomes through secondary appraisal, resilience had a direct negative impact on maladaptive outcomes. Although the precise process through which the resilience factors influence the outcomes of stress is by no means conclusive, it was postulated that the direct effect of resilience on maladaptive outcomes may reflect more effective and adaptive coping strategies in dealing with stress. For example, coping flexibility facilitates the use of more varied coping strategies and augments responsiveness and attentiveness to the perceived demands of the situation (Hong \& Chang, 2002); and emotional self-regulation may well 
foster a sense of self-efficacy in terms of controlling oneself, which would diminish negative psychological and emotional reactions to stress (Yamaguchi, 2001).

Implications

The findings of the present study have several implications. At a conceptual level, it provides elucidation of the nature of occupational stress and the relationships between person and cognitive determinants of stress and stress outcomes. In addition, it highlights the importance of including person factors in order to derive a more comprehensive formulation of the occupational stress process. Monroe and Kelly (1997) suggest that an understanding of the antecedents of appraisal enables investigators to make more penetrating statements about the etiological mechanisms or role of appraisal in the stress process (Lazarus \& Folkman, 1984). Fundamentally, appraisal may be a reflection of underlying forces that are more directly responsible for incurring susceptibility rather than a determinant of such susceptibility itself. The inclusion of other elements in the model is required to determine the essential processes involved in producing maladaptive outcomes. Thus, by integrating measures of the antecedents and components that contribute to appraisal, one can better test competing views of the role of appraisal in the stress process (Monroe \& Kelly, 1997).

The finding that psychological resilience plays an adaptive role in the stress process has implications for personnel selection and related human resource functions. As underscored in the introduction, occupational stress has significant consequences on organizations. In addition to monetary costs accumulated through absenteeism, compensation claims, health insurance and direct medical expenses, researchers have argued that stress has more indirect effects on the organization through the impediment of innovation and creativity (e.g., Amabile et al., 1996), job performance (e.g., Motowildo et al., 1986), organizational citizenship (e.g., Kruse, 1995), and counterproductive behaviors (e.g., Jones \& Boye, 1992). This makes the selection of individuals who are able to adapt to varied stressful encounters more essential, especially in the context of the global work environment. The present study lends preliminary credence to the use of selection tests based on the posited resilience factors.

The relevance of resilience in occupational stress can also be extended to secondary interventions directed at the reduction of organizational stress. Such interventions focus on learning and development (L\&D) programs at the individual level to alleviate the impact that stressors exert on employees, rather than making changes to the organizational environment (Dewe, 1994). They are targeted at individual rather than organizational changes, and they aim primarily to increase individuals' awareness of their levels of strain and to enhance personal coping strategies. 
The current findings advocate the use of cognitive techniques to aid individuals in dealing with work stress. The implication is the use of such techniques to help individuals appraise potential work stressors more constructively, thus minimizing aversive or maladaptive responses. In addition, proactive or preventive interventions could enhance individuals' levels of resilience through skills training, which would possibly promote more adaptive stress management.

\section{Limitations}

There are several limitations to the present study that must be acknowledged. First, although the study used actual job incumbents in a naturalistic context, the results may not be generalizable to other organizational populations given that incumbents from only a single organization were utilized. It is possible that variations in organizational climates and contexts of the structure and processes may yield dissimilar results.

Second, it is noteworthy that only a cross-section of the dynamic and cyclical stress process was investigated. Mack, Nelson, and Quick's (1998) dynamic process stress model depicts a cyclical process of stress in which coping behaviors influence the perception stage. It proposes a reciprocal relationship between coping and appraisal. The outcomes of coping can bring about changes and alterations to the perceived stressor, which will be reassessed by the individual, thus giving rise to a repetitive sequence of appraisal and feedback until the encounter is resolved. Similarly, transactional theory (Lazarus \& Folkman, 1984) posits that the person and the environment are in a dynamic relationship that is constantly changing. This means that appraisals are likely to change throughout a stressful encounter as a result of shifts in the person-environment interaction due to new information from the environment and/or coping efforts (Lazarus \& Folkman, 1984).

To the extent that participant responses to cognitive appraisals and outcomes of the stressor were collected for an event that was already being experienced by the respondents for a period of time, the cyclical processes of appraisal and coping were already in progress. Therefore, assessment of appraisals and outcomes was on the basis of a single phase of the cyclical dynamic stress process.

\section{Future Research}

The central focus of occupational stress research has tended towards the influence of situational or cognitive factors in the stress process with the exclusion of person factors. This paper has proposed the construct of psychological resilience in the Asian context and found that it affects maladaptive outcomes of stress both directly as well as indirectly through secondary appraisals of control. It was posited that the resilience factors 
influence secondary appraisal through the perceptual lens of pre-existing expectation, and influence stress outcomes through the promotion of more adaptive coping strategies. Future research should examine and attempt to clarify the precise process through which the resilience factors impinge on these variables.

Future research should also look into the development of appraisal measures. The Stress Appraisal Measure (Peacock \& Wong, 1990) used in the present study is one of the few multiple-item instruments designed specifically to assess dimensions of primary and secondary appraisal, which explicitly distinguishes coping from appraisal processes (Monroe \& Kelley, 1997). Since measurement is an essential component for construct exploration and validation (Loevinger, 1957), investigators need to devote more extensive research and devise additional approaches to the measurement of appraisal in order to advance theory on stress and appraisal (Monroe \& Kelley, 1997).

Another interesting research direction concerns the involvement of coping in the occupational stress process and its relationships with the resilience factors. As previously suggested, one way in which the resilience factors might influence maladaptive outcomes is through the promotion of more constructive and adaptive coping strategies. Both primary and secondary appraisals of a stressful encounter may also influence coping, which, in turn, may influence outcomes of stress. For example, appraisals of uncontrollability and threat may predict more emotion-focused coping due to accompanying negative emotions which may interfere with problem-focused forms of coping, while perceptions of control and challenge, which are not accompanied by such negative reactions, may predict more problem-focused forms of coping (Folkman, 1984).

Finally, it should be noted that a clear conceptual separation of coping efforts from cognitive appraisals is necessary if the coping construct is to be used to predict outcome (Folkman \& Lazarus, 1980). Folkman (1984) argues that many coping strategies can have an appraisal function in that they shape the meaning or significance of an event, and conversely, many forms of appraisal can have a coping function in that they help regulate distress. Future research should clarify conceptual and theoretical distinctions between coping and appraisal processes and explicate the relationships between coping, resilience, appraisal, and stress, with particular consideration for the context in which they occur.

\section{References:}

Abramson, L. Y., Seligman, M. E. P., \& Teasdale, J. D. (1978). Learned helplessness in humans: Critique and reformulation. Journal of Abnormal Psychology, 87, 49-74. 
Alfredsson, L., \& Theorell, T. (1983). Job characteristics of occupations and myocardial infection risk: Effect of possible confounding factors. Social Science and Medicine, 17, 1497-1503.

Amabile, T. M., Conti, R., Coon, H., Lazenby, J., \& Herron, M. (1996). Assessing the work environment for creativity. Academy of Management Journal, 39, 1154-1184.

Atkinson, M., \& Violato, C. (1994). Neuroticism and coping with anger: The trans-situational consistency of coping responses. Personality and Individual Differences, 17, 769-782.

Beeghly, M., \& Chicchetti, D. (1994). Child maltreatment, attachment and the self system: Emergence of an internal state lexicon in toddlers at high social risk. Development and Psychopathology, 6, 5-30.

Bentler, P.M. (1990). Comparative fit indexes in structural models. Psychological Bulletin, 107, 238-246.

Bentler, P. M., \& Bonett, D. G. (1980). Significance tests and goodness of fit in the analysis of covariance structures. Psychological Bulletin, 88, 588-606.

Bollen, K. A. (1989). Structural equations with latent variables. New York: Wiley.

Bond, M. H., \& Tornatzky, L. G. (1973). Locus of control in students from Japan and the United States: Dimensions and levels of response. Psychologia, 16, 209-213.

Cartwright, S., \& Cooper, C. (1990). The impact of mergers and acquisitions on people at work: Existing research and issues. British journal of Management, 1, 65-76.

Carver, C. S., Scheier, M. F., \& Weintraub, J. K. (1989). Assessing coping strategies: A theoretically based approach. Journal of Personality and Social Psychology, 56, 267-283.

Chang, W. C. (1985). A cross-cultural study of depressive symptomatology. Culture, Medicine and Psychiatry, 9, 295-317.

Chang, W. C., Chu, R., Tong, E. M. W., \& Koh, J. B. K. (2002). Asian subjective well-being scale: A measure of subjective well-being in a collectivist context. Manuscript in preparation, National University of Singapore, Singapore.

Chang, W. C., Chua, W. L., \& Toh, Y. (1997). The concept of psychological control in the Asian context. In K. Leung, U. Kim, S. Yamaguchi, \& Y. Kashima (Eds.), Progress in Asian Social Psychology, Vol. 1 (pp. 95-117). Singapore: Wiley.

Chang, W. C. (2007). Forged in fire: Indigenous resilience factors of Singaporean Chinese and Malays. Advances in Asian Social Psychology, 4, 17-39. 
Chang, W. C. \& Lim, L. H., (2007). Resilience and Subjective Wellbeing in an Asian Context: Singapore. Journal of Psychology in the Chinese Societies, 8(1), 47-69.

Choi, I., \& Nisbett, R. E. (1998). Situational salience and cultural differences in the correspondence bias and in the actor-observer bias. Personality and Social Psychology Bulletin, 24, 949-960.

Cohen, F., \& Lazarus, R. S. (1973). Active coping processes, coping dispositions, and recovery from surgery. Psychosomatic Medicine, 35, 375389.

Cowen, E. L., Wyman, P. A., Work, W. C., Kim, J. Y., Fagen, D. B., \& Magnus, K. B. (1997). Follow-up study of young stress-affected and stressresilient urban children. Development and Psychopathology, 9, 564-577.

Cross, S. E. (1995). Self-construals, coping, and stress in cross-cultural adaptation. Journal of Cross-Cultural Psychology, 26, 673-697.

David, P. J., \& Suls, J. (1999). Coping efforts in daily life: Role of big five traits and problem appraisals. Journal of Personality, 67, 265-194.

Dewe, P. (1994). EAPs and stress management: From theory to practice to comprehensiveness. Personnel Review, 23, 21-32.

Dweck, C. S. (2000). Self-theories: Their role in motivation, personality and development. Philadelphia, PA: Psychological Press.

Folkman, S. (1984). Personal control and stress and coping processes: A theoretical analysis. Journal of Personality and Social Psychology, 46, 839852.

Folkman, S., \& Lazarus, R. S. (1980). An analysis of coping in a middleaged community sample. Journal of Health and Social Behavior, 21, 219239.

Folkman, S., Lazarus, R. S., Dunkel-Schetter, C., Delongis, A., \& Gruen, R. J. (2001). The dynamics of a stressful encounter. In E. T. Higgins, \& A. W. Kruglanski (Eds.), Motivational science: Social and personality perspectives (pp. 111-127). Philadelphia: Psychology Press.

Garmezy, N. (1991). Resilience in children's adaptation to negative life events and stressed environments. Pediatrics, 20, 459-466.

Goh, Y. W. (2003). Occupational stress and coping: A multi-dimension ecological perspective. Doctoral dissertation. University of Queensland, Australia.

Heine, S. J. (2001). Self as cultural product: An examination of East Asian and North American selves. Journal of Personality, 69, 881-905.

Heine, S. J., \& Lehman, D. R. (1995). Cultural variation in unrealistic optimism: Does the West feel more invulnerable than the East? Journal of Personality and Social Psychology, 72, 1268-1283. 
Heine, S. J., Leman, D. R., Markus, H. R., \& Kitayama, S. (1999). It there a universal needs for positive self-regards? Psychological Review, 106, 766794.

Heine, S. J., Takata, T., \& Lehman, D. R. (2000). Beyond self-presentation: Evidence for self-criticism among Japanese. Personality and Social Psychology Bulletin, 26, 71-78.

Holahan, C. J., \& Moos, R. H. (1987). Risk, resistance and psychological distress: A longitudinal analysis with adults and children. Journal of Abnormal Psychology, 96, 3-13.

Hong, R. Y., \& Chang, W. C. (2002). Coping flexibility: conceptualization and measurement. Unpublished manuscript, National University of Singapore, Singapore.

Howard, A. (1995). A framework for work change. In A. Howard (Ed.), The changing nature of work (pp. 3-44). San Francisco: Jossey-Bass.

Jones, J. W., \& Boye, M. W. (1992). Job stress and employee counterproductivity. In J. C. Quick, J. J. Hurrell, \& L. R. Murphy (Eds.), Stress and well-being at work (pp. 239-251). Washington, DC: American Psychological Association.

Joreskog, K., \& Sorbom, D. (1986). LISREL 6: Analysis of linear structural relationships by maximum likelihood and least square methods. Mooresville, IN: Scientific Software.

Joreskog, K., \& Sorbom, D. (1989). LISREL 7: A guide to the program and applications (2nd ed.). Chicago: SPSS.

Joreskog, K., \& Sorbom, D. (1993). LISREL 8: Structural equation modeling with the SIMPLIS command language. Hillsdale, NJ: Lawrence Erlbaum Associates, Inc.

Karasek, R., \& Theorell, T. (1990). Healthy work: Stress, Productivity and the reconstruction of working life. New York: John Wiley \& Sons.

Kawanishi, Y. (1995). The effects of culture on beliefs about stress and coping: Causal attribution of Anglo-American and Japanese persons. Journal of Contemporary Psychotherapy, 25, 49-60.

Kim, U. \& Park, Y. S. (1998). Factors influencing stress and life-satisfaction level of Korean adolescents: Comparison of rural and urban students. Korean Journal of Health Psychology, 3, 79-101.

Kinicki, A. J., \& Latack, J. C. (1990). Explication of the construct of coping with job loss. Journal of Vocational Behavior, 36, 339-360.

Kitayama, S., Markus, H. R., Matsumoto, H., \& Norasakkunkit, V. (1997). Individual and collective processes of self-esteem management: Selfenhancement in the United States and self-depreciation in Japan. Journal of Personality and Social Psychology, 72, 1245-1267. 
Kruse, B. G. (1995). Affective and cognitive mediation of the relationship situational constraints and organizational citizenship behaviors. Unpublished master's thesis, Central Michigan University, Michigan.

Lazarus, R. S. (1966). Psychological stress and the coping process. New York: Oxford University Press.

Lazarus, R. S. (1991). Emotion and adaptation. New York: Oxford University Press.

Lazarus, R. S., \& Folkman, S. (1984). Stress, appraisal, and coping. New York: Springer.

Loevinger, J. (1957). Objective tests as instruments of psychological theory. Psychological Reports, 3, 635-694.

Luthar, S. S. (1999). Poverty and children's adjustment. Newbury Park, CA: Sage.

Mack, D. A., Nelson, D. L., \& Quick, J. C. (1998). The stress of organizational change: A dynamic process model. Applied Psychology: An International Review, 47, 219-232.

Markus, H. R., \& Kitayama, S. (1991). Culture and the self: Implications for cognition emotion, and motivation. Psychological Review, 98, 224-253.

Masten, A., \& Garmezy, N. (1985). Risk, vulnerability, and protective factors in developmental psychopathology. In B. Lahey \& A. Kazdin (Eds.), Advances in clinical child psychology Vol. 8 (pp. 1-52). New York: Plenum Press.

McCrae, R. R. (1989). Age differences and changes in the use of coping mechanisms. Journal of Gerontology, 44, 161-169.

McGrath, J. E. (1970). Social and psychological factors in stress. New York: Holt, Rinehart, \& Winston.

Meijer, Z. Y., Hiene, S. J., \& Yamaguchi, M. (1999). Remember those good old days? Culture, self-discrepancies, and biographical memory. Symposium presentation at the 3rd Conference of the Asian Association of Social Psychology, Taipei, Taiwan.

Meyer, G. J., \& Shack, J. R. (1989). Structural convergence of mood and personality: Evidence for old and new directions. Journal of Personality and Social Psychology, 57, 691-706.

Monroe, S. M., \& Kelley, J. M. (1997). Measurement of stress appraisal. In S. Cohen, R. C. Kessler, \& L. U. Gordon (Eds.), Measuring stress: A guide for health and social scientists (pp. 122-147). New York: Oxford University Press.

Moos, R. H., \& Shaefer, J. A. (1993). Coping resources and processes: Current concepts and measures. In L. Goldberger \& S. Breznitz (Eds.), Handbook of stress: Theoretical and clinical aspects (pp. 234-257). New York: Free Press. 
Motowildo, S. J., Packard, J. S., \& Manning, M. R. (1986). Occupational stress: Its causes and consequences for job performance. Journal of Applied Psychology, 71, 618-629.

Neo, A. H. C., Koh, J. B. K., \& Chang, W. C. (2002). Alternation or fusion: Singaporean Chinese bilinguals and their social attitudes. Paper presented at the International Conference on Language and Social Behavior, Hong Kong.

Noe, R., \& Ford, K. J. (1992). Emerging issues and new directions for training research. Research in Personnel and Human Resource Management, $10,345-384$.

O’Brien, T. B. \& DeLongis, A. (1996). The interactional context of problem, emotion- and relationship-focused coping: The role of the big five personality factors. Journal of Personality, 64, 775-813.

O’Dougherty-Wright, M., Masten, A. S., Northwood, A., \& Hubbard, J. J. (1997). Long term effects of massive trauma: Developmental and psychobiological perspectives. In D. Cicchetti \& S. L. Toth (Eds.), Rochester symposium on developmental psychopathology: Vol. 8. Developmental perspectives on trauma (pp. 181-225). Rochester, NY: University of Rochester Press.

Peacock, E. J., \& Wong, P. T. P. (1990). The stress appraisal measure: A multidimensional approach to cognitive appraisal. Stress Medicine, 6, 227236.

Peterson, C., \& Seligman, M. E. P. (1984). Causal explanations as a risk factor for depression: Theory and evidence. Psychological Review, 91, 347374.

Rothbaum, F., Weisz, J. R., \& Snyder, S. S. (1982). Changing the world and changing the self: A two-process model of perceived control. Journal of Personality and Social Psychology, 42, 5-37.

Schaubroeck, J., \& Merritt, D. (1997). Divergent effects of job control on coping with work stressors: The key role of self-efficacy. Academy of Management Journal, 40, 738-754.

Seligman, M. E. P., Abramson, L. Y., Semmel, A., \& Von Baeyer, C. (1979). Depressive attributional style. Journal of Abnormal Psychology, 88, 242-247.

Spector, P. (1986). Perceived control by employees: A meta-analysis of studies concerning and participation at work. Human Relations, 39, 10051016.

Spielberger, C. D., Gorsuch, R. L., \& Lushene, R. D. (1970). STAI: Manual for the State-Trait Anxiety Inventory. Palo Alto: Consulting Psychologists Press. 
Steiger, J. H. (1990). Structural model evaluation and modification: An interval estimation approach. Multivariate Behavioral Research, 25, 173180.

Suls, J., David, J. P., \& Harvey, J. H. (1996). Personality and coping: Three generations of research. Journal of Personality, 64, 711-735.

Terry, D. J. (1994). Determinants of coping: The role of stable and situational factors. Journal of Personality and Social Psychology, 66, 895910.

Vecchio, R., Hearm, G., \& Southey, G. (1996). Organizational behavior. Sydney: Harcourt Brace.

Watson, D., Clark, L. A. \& Tellegen, A. (1988). Development and validation of brief measures of positive and negative affect: The PANAS scales. Journal of Personality and Social Psychology, 54, 1063-1070.

Watson, D., \& Hubbard, B. (1996). Adaptational style and dispositional structure: Coping in the context of the five-factor model. Journal of Personality, 64, 737-774.

Werner, E. E., Bierman, J. M., \& French, F. E. (1971). The children of Kauai Honolulu. Hawaii: University of Hawaii Press.

Werner, E. E., \& Smith, R. (1992). Overcoming the odds: High risk children from birth to adulthood. Ithaca, NY: Cornell University Press.

Yamaguchi, S. (2001). Culture and control orientations. In D. Matsumoto (Ed.), The handbook of culture and psychology (pp. 223-244). New York: Oxford University Press.

Zung, W. W. K. (1965). A self-rating depression scale. Archives of General Psychiatry, 12, 63-70.

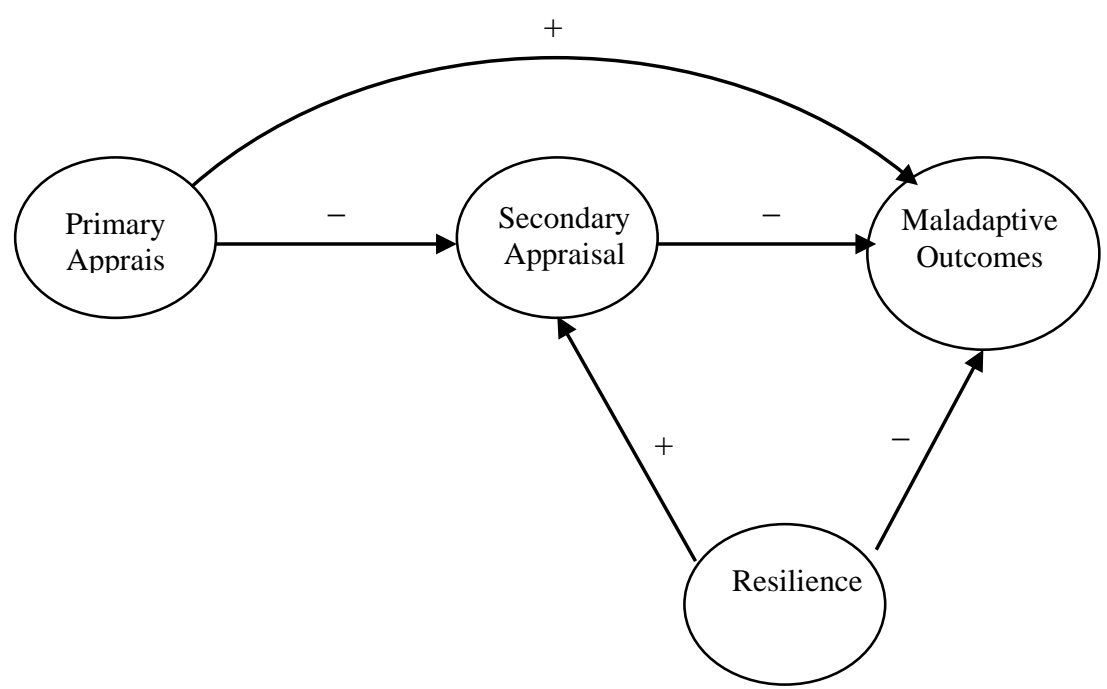

Figure 1. Hypothesized model of resilience in the occupational stress process. 
Table 1 Means, Standard Deviations, Correlations, and Reliabilities Among Indicator Variables $(\mathrm{N}=182)$

\begin{tabular}{|c|c|c|c|c|c|c|c|c|c|c|c|c|c|c|c|}
\hline \multicolumn{16}{|c|}{ Indicator } \\
\hline & Variables & $\underline{\mathrm{M}}$ & $\underline{\mathrm{SD}}$ & 1 & 2 & 3 & 4 & 5 & 6 & 7 & 8 & 9 & 10 & 11 & 12 \\
\hline 1. & Thr & 13.99 & 2.94 & .88 & & & & & & & & & & & \\
\hline 2. & Cen & 13.12 & 3.11 & $.70 *$ & .91 & & & & & & & & & & \\
\hline 3. & Cha & 8.75 & 2.06 & $-.52 *$ & $-.51 *$ & .79 & & & & & & & & & \\
\hline 4. & Cs & 12.91 & 3.67 & $-.28 *$ & $-.28 *$ & $.22 *$ & .86 & & & & & & & & \\
\hline 5. & Co & 11.27 & 3.22 & $-.29 *$ & $-.23 *$ & $.25 *$ & $.44^{*}$ & .85 & & & & & & & \\
\hline 6. & Nc & 13.13 & 3.29 & $.33 *$ & $.39 *$ & $-.36^{*}$ & $-.47^{*}$ & $-.53 *$ & .89 & & & & & & \\
\hline 7. & Anx & 36.64 & 10.75 & $.35 *$ & $.40 *$ & $-.33 *$ & $-.54 *$ & $-.47 *$ & $.45^{*}$ & .93 & & & & & \\
\hline 8. & Dep & 45.24 & 8.36 & $.29 *$ & $.23 *$ & $-.28 *$ & $-.51^{*}$ & $-.44 *$ & $.43^{*}$ & $.66 *$ & .77 & & & & \\
\hline 9. & $\mathrm{Na}$ & 19.68 & 7.73 & $.27 *$ & $.28 *$ & $-.27^{*}$ & $-.52 *$ & $-.36^{*}$ & $.46^{*}$ & $.54 *$ & $.47 *$ & .94 & & & \\
\hline 10. & Inc & 30.93 & 4.41 & -.12 & -.03 & -.06 & $.31^{*}$ & $.21 *$ & $-.23 *$ & $-.41 *$ & $-.37 *$ & $-.32 *$ & .75 & & \\
\hline 11. & Esr & 30.18 & 4.94 & -.11 & -.10 & .09 & $.34 *$ & $.23 *$ & $-.23 *$ & $-.58 *$ & $-.43 *$ & $-.39 *$ & $.52 *$ & .82 & \\
\hline 12. & Cf & 46.99 & 6.08 & -.06 & -.01 & .03 & $.16 *$ & $.22 *$ & $-.28 *$ & $-.33 *$ & $-.34 *$ & $-.20 *$ & $.40^{*}$ & $.49 *$ & .90 \\
\hline
\end{tabular}

Note. $\quad$ Thr = Threat; Cen = Centrality; Cha = Challenge; Cs = Controllable by Self; Co = Controllable by Others; Uc = Uncontrollable by Anyone; Anx = Anxiety; Dep = Depression;

$\mathrm{Na}=$ Negative Affectivity; Inc = Incremental Implicit Belief About Self; Esr = Emotional

Self-Regulation; Cf = Coping Flexibility. Cronbach’s Alpha estimates of reliabilities are on the diagonal.

$* \mathrm{p}<.05$

Table 2 Model Fit Indices for Structural Equation Models Estimated

\begin{tabular}{|c|c|c|c|c|c|c|c|c|c|c|}
\hline $\begin{array}{c}\text { Model } \\
\text { Specification }\end{array}$ & $\underline{\mathrm{df}}$ & $\chi^{2}$ & $\Delta \underline{\mathrm{df}}$ & $\Delta \chi^{2}$ & AGFI & NNFI & CFI & IFI & RMSEA & SRMSR \\
\hline $\begin{array}{l}\text { Hypothesized } \\
\text { model }\end{array}$ & 49 & 77.78 & & & .90 & .95 & .97 & .97 & .05 & .06 \\
\hline $\begin{array}{c}\text { Alternative } \\
\text { model }^{\mathrm{a}}\end{array}$ & 48 & 75.68 & 1 & 2.10 & .90 & .95 & .97 & .97 & .05 & .04 \\
\hline $\begin{array}{l}\text { Note. AGFI } \\
\text { comparative fi } \\
\text { approximation; } \\
\text { a Same as hyp } \\
\text { estimated. } \\
\text { * } p<.05\end{array}$ & $\begin{array}{l}\text { dex } \\
\text { MS } \\
\text { esiz }\end{array}$ & $\begin{array}{l}\text { ted g } \\
\text { IFI }= \\
=\text { st } \\
\text { mo }\end{array}$ & $\begin{array}{l}\text { dnes } \\
\text { ncrei } \\
\text { dardi } \\
\text { ex }\end{array}$ & enta & $\begin{array}{l}\text { index } \\
\text { fit ind } \\
\text { t mear } \\
\text { rect e }\end{array}$ & $\begin{array}{l}\text { NNFI } \\
\text {; RM } \\
\text { quare } \\
\text { ect fro }\end{array}$ & $\begin{array}{l}=\text { no } \\
\text { EA }= \\
\text { sidu } \\
\text { res }\end{array}$ & me & $\begin{array}{l}\text { fit index } \\
\text { n square } \\
\text { primary }\end{array}$ & $\begin{array}{l}\text {; CFI = } \\
\text { error of } \\
\text { appraisal }\end{array}$ \\
\hline
\end{tabular}




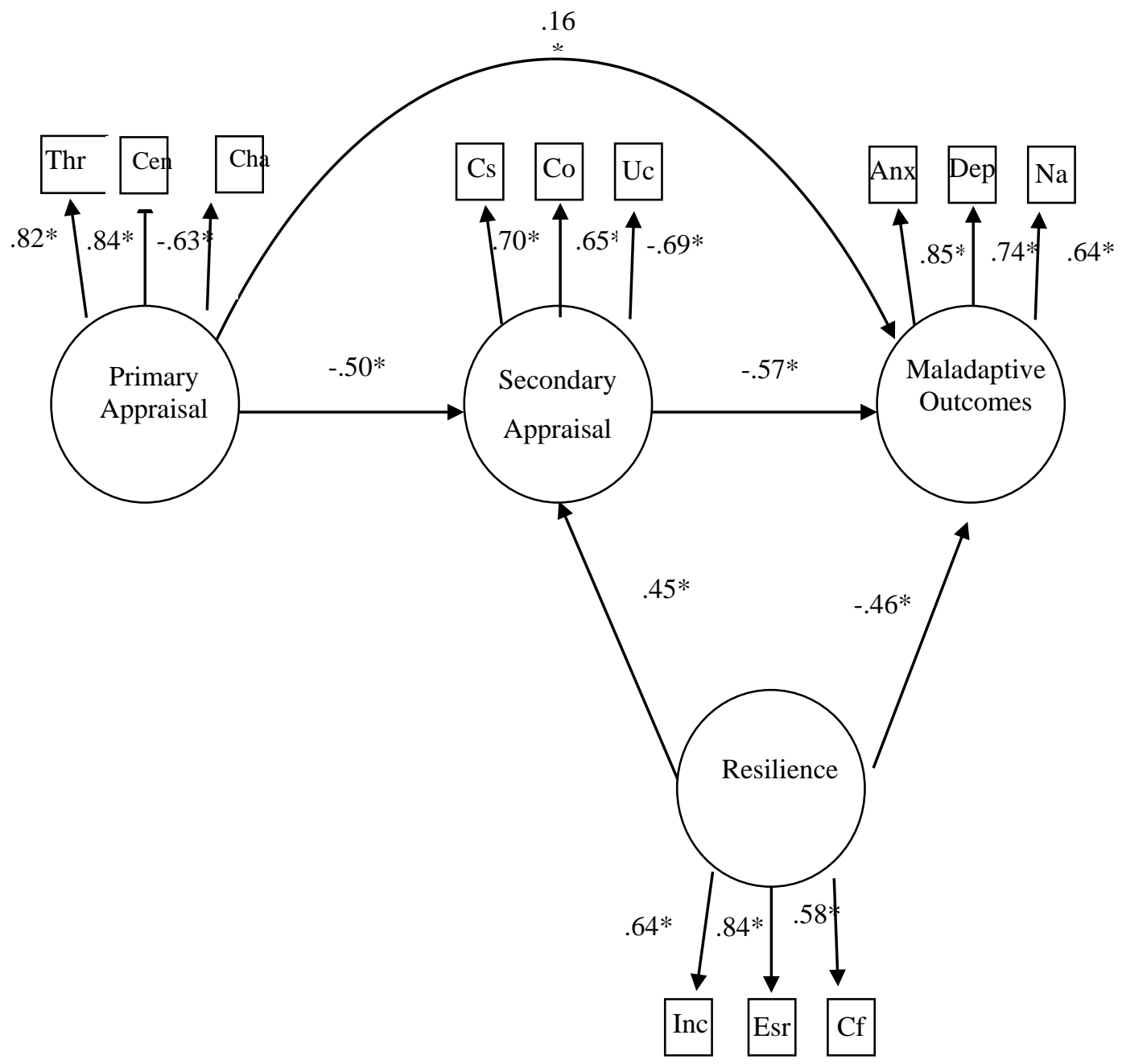

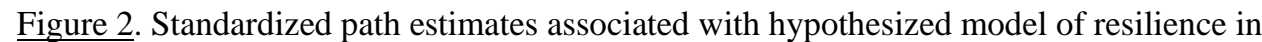
the occupational stress process.

Note. $\quad$ Thr $=$ Threat; Cen $=$ Centrality; Cha $=$ Challenge; Cs $=$ Controllable by Self; Co = Controllable by Others; Uc = Uncontrollable by Anyone; Anx = Anxiety; Dep = Depression; $\mathrm{Na}=$ Negative Affectivity; Inc = Incremental Implicit Belief About Self; Esr = Emotional Self-Regulation; $\mathrm{Cf}=$ Coping Flexibility.

$* \mathrm{p}<.05$. 